\title{
Measuring obscuration and reprocessing of AGN emission
}

\author{
N. A. Levenson ${ }^{1}$ \\ ${ }^{1}$ Department of Physics and Astronomy, University of Kentucky, Lexington, KY 40506, USA \\ email:levenson@pa.uky.edu
}

\begin{abstract}
The intrinsic emission of obscured active galactic nuclei (AGN) cannot be measured directly, but the obscured examples uniquely allow determination of the physical conditions near the central engine on 10-100 pc scales. The reprocessed radiation that emerges at X-ray and infrared energies, in particular, reveals the distribution of the obscuring medium. In general, $\mathrm{X}$-ray spectroscopy shows the total column density along the line of sight, and in extremely obscured (Compton thick) cases, the fluorescent iron $\mathrm{K} \alpha$ emission line is sensitive to viewing angle and covering fraction. Observed near- and mid-infrared spectra require an inhomogeneous distribution of material around the AGN, and they specifically depend on parameters such as the optical depth per obscuring cloud and the number of clouds along the line of sight. The total spatial extent of the obscuring region determines the shape of the far-infrared spectrum.
\end{abstract}

\section{Compton Thick AGN}

The most detailed information about the immediate circumnuclear environment of active galactic nuclei (AGN) on 10-100 pc scales comes from indirect views of the central engine at X-ray and infrared (IR) wavelengths. These combined data leave several outstanding problems, however. First, X-ray data indicate a wide range of obscuring column densities, yet the IR spectral energy distributions vary little. Second, the depth of the $10 \mu \mathrm{m}$ silicate feature is not correlated with the X-ray-determined column density. Third, the corresponding silicate emission is not observed in Seyfert 1s. We begin to solve these problems by recognizing the more complex X-ray emission of real Seyfert galaxies and developing models of infrared emission that include inhomogeneous obscuration.

When the column density $N_{H}>10^{24} \mathrm{~cm}^{-2}$, the AGN is not directly detected, even up to $8 \mathrm{keV}$. In the obscuring medium, the intrinsic emission is strongly reprocessed, emerging with an effective flat photon index and diminished intensity, typically around $1 \%$ of the intrinsic source (Figure 1, left). In these Compton thick AGN, the large equivalent width $(\mathrm{EW})$ of the $\mathrm{Fe} \mathrm{K} \alpha$ line at $6.4 \mathrm{keV}$ can be an effective diagnostic of the geometry of the obscuring medium (Krolik et al.1994, Levenson et al.2002). An EW > $2 \mathrm{keV}$, for example, requires an opening angle $\Theta \leqslant 20^{\circ}$, or equivalently, a covering fraction greater than $90 \%$. Furthermore, the Fe line luminosity also yields an estimate of the AGN bolometric luminosity, especially with the additional constraint of the geometry. In Circinus we find the intrinsic $2-10 \mathrm{keV}$ luminosity $L_{2-10 \mathrm{keV}}=1.8 \times 10^{42} \mathrm{erg} \mathrm{s}^{-1}$. These results from low-resolution spectroscopy of Circinus shown here are confirmed with higher-energy and high spectral resolution observations (Matt et al.1999, Sambruna et al.2001).

\section{Clumpy Obscuration Models}

A toroidal distribution of material around the central engine broadly accounts for differences between Seyfert 1s and 2s in terms of viewing geometry, and an inhomogeneous 

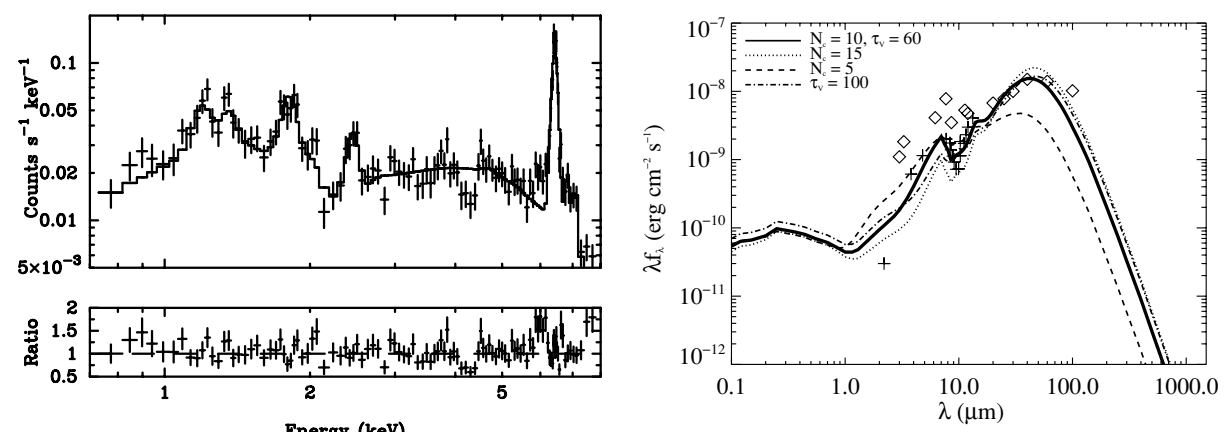

Figure 1. (left) Chandra spectrum of Circinus, a Compton thick AGN. Only indirect reprocessed emission is detected. The Fe $\mathrm{K} \alpha$ and other photoionized lines are prominent. (right) Infrared data and models of Circinus. Measurements from small apertures are marked as crosses and better represent the obscuring medium that is modeled. Large aperture measurements are plotted as diamonds. The basic model has $N_{C}=10, \tau_{v}=60$, and $i=60^{\circ}$. This model replicates the steepness of the near-IR spectrum without overpredicting the emission longward of $60 \mu \mathrm{m}$. Variations of this basic model are also plotted. Data are from Maiolino et al. (1998), Roche et al. (1991), and Sturm et al. (2000).

medium addresses the problems noted above. without severely limiting the possible variation of intrinsic AGN luminosity and torus size, as homogeneous obscuration models do. Nenkova et al. (2002) have modeled radiative transfer in these clumpy distributions, which we apply to Circinus (Figure 1, right). The emergent emission is a function of $N_{C}$, the average number of clouds along the line of sight, and $\tau_{V}$, the optical depth per cloud.

This obscuring model does not account for stellar emission at all, which is certainly significant in Circinus. A dusty starburst adds to the far-IR emission, especially on large scales. The model $N_{H}$ is lower than the value measured in X-rays. Maser emission has been observed in a warped disk around the AGN (Greenhill et al.2003), suggesting that the disk material increases the total column density. Also, a mix of cloud optical depths may be necessary to reproduce the depth of the $10 \mu \mathrm{m}$ absorption.

\section{Conclusions}

The emergent X-ray and IR spectra of obscured AGN depend on the distribution of the intervening material. X-ray observations determine column density along a single line of sight, and in Compton thick cases, the Fe K $\alpha$ line EW strongly constrains the obscurer's geometry. All IR emission is reprocessed. While further refinements of the IR models are needed, allowing for an inhomogeneous medium is essential.

This work is supported by National Science Foundation CAREER award AST-0237291.

\section{References}

Greenhill, L. J., et al. 2003, ApJ, 590, 162

Krolik, J. H., Madau, P., \& Zycki, P. T. 1994, ApJ, 420, L57

Levenson, N. A., et al. 2002, ApJ, 573, L81

Maiolino, R., Krabbe, A., Thatte, N., \& Genzel, R. 1998, ApJ, 493, 650

Matt, G., et al. 1999, A\&A, 341, L39

Roche, P. F., Aitken, D. K., Smith, C. H., \& Ward, M. J. 1991, MNRAS, 248, 606

Sambruna, R. M., et al. 2001, ApJ, 546, L13

Sturm, E., et al. 2000, A\&A, 358, 481 